Arts \& Health

An International Journal for Research, Policy and Practice

ISSN: 1753-3015 (Print) 1753-3023 (Online) Journal homepage: https://www.tandfonline.com/loi/rahe20

\title{
Determining the effects of therapeutic clowning on nurses in a children's rehabilitation hospital
}

Stefanie Blain, Shauna Kingsnorth , Lindsay Stephens \& Patricia McKeever

To cite this article: Stefanie Blain, Shauna Kingsnorth, Lindsay Stephens \& Patricia McKeever (2012) Determining the effects of therapeutic clowning on nurses in a children's rehabilitation hospital, Arts \& Health, 4:1, 26-38, DOI: 10.1080/17533015.2011.561359

To link to this article: https://doi.org/10.1080/17533015.2011.561359

册 Published online: 13 Jun 2011.

Submit your article to this journal $\sqsubset$

Lll Article views: 310

Q View related articles $\asymp$

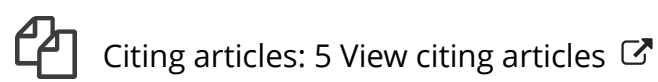




\title{
Determining the effects of therapeutic clowning on nurses in a children's rehabilitation hospital
}

\author{
Stefanie Blain $^{\mathrm{a} *}$, Shauna Kingsnorth ${ }^{\mathrm{b}}$, Lindsay Stephens ${ }^{\mathrm{c}}$ and Patricia McKeever ${ }^{\mathrm{b}, \mathrm{d}}$ \\ ${ }^{a}$ University of Michigan, Physical Medicine and Rehabilitation, Ann Arbor, MI, USA; ${ }^{b}$ Holland \\ Bloorview Kids Rehabilitation Hospital, Bloorview Research Institute, Toronto, Ontario, Canada; \\ ${ }^{c}$ University of Toronto, Department of Geography and Program in Planning, Toronto, Ontario, \\ Canada; ${ }^{d}$ University of Toronto, L.S. Bloomberg Faculty of Nursing, Toronto, Ontario, Canada
}

(Received 20 December 2010; final version received 31 January 2011)

\begin{abstract}
Objective: This pilot study aimed to determine the effects of therapeutic clowns for hospitalized children on their nurses. Methods: Semi-structured interviews with 13 nurses explored perceptions of the clowns. Measures of physiological arousal, emotion and anxiety were also obtained from nine of these 13 nurses under two conditions - the presence or absence of therapeutic clowns on the unit. Results: Physiologically, the automatic nervous system signals of eight of the nine nurses exhibited consistent changes in patterns of responding when the clowns were present. Emotionally, nurses' reports of negative mood states were also reduced; no changes in anxiety were found. Qualitative data corroborated these findings and suggest that although therapeutic clowns expressly work with children, they also have a relational impact. Conclusions: Clowning seems to improve communication, role negotiation and the meaning of the unit as a social and physical place. A recommendation for mixed method research designs is offered to determine varied effects of arts-based interventions on stakeholders within healthcare settings.
\end{abstract}

Keywords: arts-based interventions; pediatrics; nurses; rehabilitation; therapeutic clowns

\section{Background}

For centuries, art and healing have been inextricably related to one another (Dose, 2006; Society for Arts in Healthcare [SAH], 2009; Stuckey \& Nobel, 2010). Increasingly, healthcare administrators and designers have tried to exploit this relation; the arts figure in the aesthetic design of healthcare spaces and in the delivery of many interventions (Dose, 2006; Macnaughton, White \& Stacy, 2005; SAH, 2009). There is a growing body of evidence supporting the use of art, arts-based interventions and art therapy to improve a range of health-related outcomes (Daykin, Byrne, Soteriou \& O'Connor, 2008; Gold, Solli, Kruger \& Lie, 2009; Gold, Voracek \& Wigram, 2004; Goyal \& Keightley, 2008; Joronen, Rankin \& Astedt-Kurki, 2008; Maratos, Gold, Wang \& Crawford, 2008; Stuckey \& Nobel, 2010; Treurnicht Naylor, Kingsnorth, Lamont, McKeever \& Macarthur, 2010; Wood, Molassiotis \& Payne, 2010).

While work to date predominantly has explored the effects of arts-based interventions on patients (Bertini, Isola, Paolone \& Curcio, 2010; Brice \& Barclay, 2007; Chapman, Morabito, Ladakakos, Schreier \& Knudson, 2007; Hilliard, 2007) and family caregivers

*Corresponding author. Email: sblain@umich.edu 
(Costa Fernandes \& Arriaga, 2010; Rothieaux, 1997; Vagnoli, Caprilli, Robiglio \& Messeri, 2005; Walsh, Martin \& Schmidt, 2004), there is some evidence suggesting that they may affect healthcare professionals (Daykin et al., 2008; Repar \& Patton, 2007). The potential for indirect or secondary effects of the arts has been relatively unexplored. Some research suggests that the presence of the arts in healthcare may lead to enhanced nursepatient communication (Ferszt, Massotti, Williams \& Miller, 2000; Wikstrom, 2003) and positive changes in hospital environments (Francani, Zilioli, Silva, Sant'ana \& de Lima, 1998; Gatti \& da Silva, 2007; Higueras et al., 2006; Linge, 2008) resulting in higher job satisfaction (KCI Research \& Evaluation, 2002; Francis, Willis \& Garvey, 2003, cited in Daykin et al., 2008) and improved work performance (Gatti \& da Silva, 2007). Such effects may have the potential to improve morale and reduce stress thereby increasing job retention and recruitment, and improving the quality of service delivery (SAH, 2009).

Among members of rehabilitation teams, especially in complex continuing care and rehabilitation hospital settings, nurses are most consistently exposed to bedside arts-based interventions and aesthetic design features because of their around-the-clock presence and primary responsibility in monitoring the health status of patients. Furthermore, many of the outcomes targeted by therapeutic artists are relevant to nursing roles; nursing work is highly stressful and involves significant risk of compassion fatigue (Abrahamson, 2008; Joinson, 1992; McVicar, 2003; Phillips, 1996). The tasks involved in meeting the emotional and physical needs of disabled and/or ill patients and their families can be inimical to the emotional and physical health of nurses (Repar \& Patton, 2007).

The current study sought to examine the effects of one arts-based intervention therapeutic clowning - on nurses in a Canadian pediatric rehabilitation hospital. Therapeutic clowns are trained artists who use clowning to empower patients through opportunities for emotional expression, social interaction and personal control (Koller \& Gryski, 2008). Studies have shown that clown interventions have the ability to decrease anxiety and pain, and positively elevate mood in hospitalized children and their family caregivers (Battrick \& Weaver, 2007; Bertini et al., 2010; Fernandes \& Arriaga, 2010; Golan, Tighe, Dobija, Perel \& Keidan, 2009; Kingsnorth, Blain \& McKeever, 2010; Vagnoli et al., 2005). Within this literature, anecdotal and/or survey evidence indicates that clowning interventions may have secondary positive effects (de Lima, Azevedo, Nascimento \& Rocha, 2009; Linge, 2008; Oppenheim, Simonds \& Hartmann, 1997; The Northern Hospital, 2005). An evaluation of clowning in the USA reported reductions in routine stress and improved working conditions among emergency department healthcare providers (The Northern Hospital, 2005). Other studies suggest that the presence of clowns can help providers understand children outside of their role as hospitalized patients (Oppenheim et al., 1997), encourage capacity for play and expression through parody (de Lima et al., 2009; Linge, 2008), and thereby enhance communication and caregiverpatient interactions, further supporting the potential for wide-reaching impact. Based on existing evidence, we hypothesized that nurses would demonstrate positive differential physiological, emotional and behavioural responses when therapeutic clowns interacted with children in a long-term healthcare setting.

\section{Research Approach}

Given the exploratory nature of this pilot study, a multi-pronged mixed-method approach was employed to capture the heretofore unknown effects of therapeutic clowns on nurses. Questions of interest included: what are the physiological, emotional and behavioural responses of nurses to therapeutic clowns for children? How do nurses describe the effects 
of therapeutic clowning on themselves and hospitalized children? Is there a correspondence between nurses' subjective reports and physiological responses to therapeutic clowning?

\section{Setting and Intervention}

This study was conducted within the complex continuing care and brain injury units of a children's hospital that provides long-term rehabilitative care to infants, children and youth with a range of physical, cognitive and/or developmental disabilities. As part of a standard established program, highly trained Therapeutic Clowns working in a duo-clown partnership visited the unit for approximately 60 minutes twice each week. Costumed in a red plastic nose and equipped with a multitude of skills and tools (including music and rhythm, dramatic movement, physical comedy, storytelling and role-reversal, magic tricks and games), Dr Flap and Ricky endeavor to empower children during short individualized interventions (e.g. 10-15 minutes).

\section{Participants}

Fifteen nurses (two males) volunteered to participate in this study; of this sample, 13 nurses completed qualitative interviews; nine completed quantitative data collection; and data from two were excluded due to loss to follow-up. Experience as a nurse varied with a median of six years $(\min .=<1, \max .=35)$ and a median of five years on the unit $(\min .=<1, \max .=31)$. One nurse reported no prior experience with therapeutic clowns and one nurse reported being fearful of clowns. Ethical approval was received from the hospital and written consent obtained from all participants.

\section{Study Design and Methods}

Over the course of four days, data were collected using an ABAB design (A: no intervention, B: exposure to the children's routine therapeutic clown visits) (Kingsnorth et al., 2010) in spaces such as nursing stations, patient rooms and hallways. Of note, the therapeutic clowns did not interact directly with the nurses; rather, nurses were within lineof-sight of standard interactions between children and the clowns. On each day, data were collected in a morning baseline session and in an afternoon intervention session, each lasting five minutes. The timing of the sessions was consistent to control for the effects of natural variations in circadian rhythms and hospital routines. Participating nurses were instructed to keep their caffeine consumption constant for the duration of data collection for similar reasons. As all were on active care-giving duty, restrictions related to movement and conversations were not imposed.

\section{Data Collection}

Three types of data were collected (physiological, emotional and behavioural) as described below.

Physiological measures. Four physiological signals of the autonomic nervous system (ANS) that are known to vary with levels of stress and with emotional state were collected from each participant, namely, electrodermal activity (EDA), fingertip temperature, respiration patterns, and blood volume pulse (BVP) (Blain, Chau \& Mihailidis, 2008; Picard, Vyzas \& Healey, 2001). EDA is a measure of the electrical conductance of the skin, 
which varies upon stimulation of the sympathetic nervous system; fingertip temperature is influenced by the contraction of vascular structures called ateriovenous anatomoses upon ANS stimulation; the respiratory and cardiovascular systems are innervated by and responsive to both branches of the ANS and vary their patterns upon ANS stimulation. Salient changes in any of these four physiological measures may indicate a change in emotional state or anxiety levels. To measure EDA, fingertip temperature and heart rate, three Thought Technology ${ }^{\circledR}$ sensors were secured to the fingers of participating nurses. To measure respiration, a fourth sensor was secured around their thoracic cavity. ANS data were recorded using a Biograph Infiniti ${ }^{\complement}$ data acquisition unit and downloaded after the session for offline analysis.

Emotional and behavioural measures. To elucidate the nature of changes in ANS signals and to determine change in mood and anxiety, two self-ratings measures were employed: Feeling Faces Cards ${ }^{\circledR}$ (Beder, 2004) and the State-Trait Anxiety Inventory (STAI-Form Y) (Spielberger, 1983). Prior to each session, participating nurses were asked "How do you feel right now?" and instructed to select one or more Feeling Faces Cards ${ }^{\circledR}$. These cards present 20 exaggerated facial expressions depicting mood states of ethnically diverse male and female children. The STAI was administered once daily at the completion of the afternoon session; it consists of two 20-item self-report scales designed to distinguish between state (transient) and trait (personality) anxiety.

In addition to self-reported ratings, nurses each participated in a single semistructured, open-ended interview that took place independent of the quantitative data collection periods. Participants responded to two questions. First, they were asked what they liked and did not like about the clowns' visits to patients, and what kinds of effects the clowns had on them and their patients. As these interviews took place while nurses' held active responsibilities, interview times ranged from 3-36 minutes with an average length of 12 minutes.

\section{Data Analysis}

Physiological data. Because individuals have idiosyncratic ANS responses to stimuli in their environments (Lacey \& Lacey, 1958), each of the four signals was analyzed independently for each nurse using 10 salient features extracted from raw physiological data for each collection session (see Table 1).

For each day of data collection, the difference in these features between morning and afternoon sessions was calculated. The daily differences in these signal features were compared between interventions A and B to determine whether a consistent change in physiological responding to the therapeutic clowns had occurred.

Table 1. Features Extracted from Physiological Signals Collected from Nurse Participants.

\begin{tabular}{llll}
\hline & \multicolumn{3}{c}{ Extracted features } \\
\cline { 2 - 4 } Raw ANS Signal & Feature 1 & Feature 2 & Feature 3 \\
\hline Electrodermal activity (EDA) & Number of electrodermal reactions & Average & N/A \\
Fingertip temperature & Number of significant changes in slope & Average & N/A \\
Respiration rate & Frequency & Amplitude & Average \\
Heart rate & Average direction of change & Maximum & Average \\
\hline
\end{tabular}


Emotional and behavioural data. To explore changes in emotion, mood reports were assigned either a positive or a negative valence (Kingsnorth et al., 2010). Total frequencies of positive and negative moods were summed across both days of intervention $\mathrm{A}$ and intervention B, respectively, for the morning and afternoon sessions. Due to the variability in responses, statistical analysis of the emotion data was not feasible. Differences in STAI scores on the state scale were analyzed statistically using an ANOVA to examine change within and across interventions (A and $\mathrm{B}$ ).

Qualitative interview data. The semi-structured, open-ended interviews were transcribed in full and data were analyzed for patterns, themes and narrative descriptions of the participant interactions (e.g. nurse-clown; child-clown) using NVivo8 software. A coding scheme was developed based on the project goals, research and interview questions, and themes that emerged from the interviews. The data were explored using qualitative techniques including basic content analysis, development and testing/confirming of emergent themes, and exploration of the relations between themes and participant identities (e.g. self-described fear of clowns). Once emergent themes were identified, data analysis was guided by published theoretical and/or research literature about nursing workplaces (Gilmour, 2005; McGilton, Bowers, McKenzie-Green, Boscart \& Brown, 2009) and health geography (Andrews, 2004, 2006; Kearns \& Moon, 2002; Poland, Lehoux, Holmes \& Andrews, 2005).

\section{Results}

\section{Physiological Responses}

As illustrated in Table 2, eight of nine participating nurses showed consistent physiological changes in at least two ANS signal features on days when the clowns were present with children in comparison to the days when the clowns were absent.

\section{Emotional and Behavioral Responses}

With respect to mood, the most common responses across the eight data collection sessions were reports of being content $(n=33)$ and happy $(n=28)$. Negative mood reports included being tired $(n=16)$, worried $(n=8)$, anxious $(n=8)$ or other varied emotions $(n=5)$; no expressions of fear were reported. These emotions were reported individually or in combination (e.g. "happy and content" or "anxious and tired"). Figure 1 presents frequencies of mood reports according to valence for morning and afternoon sessions on non-clown and clown days. Relative to non-clown days, a visual inspection revealed a trend towards higher frequencies of positive emotions and a marked drop in the overall frequency of negative mood reports on clown days. No significant differences in state anxiety were found $(p>.05)$, with an overall mean score of $32.26(S D=10.03)$.

With respect to the interview data, two central themes emerged: (1) the effects of therapeutic clowns on the emotional tone and environment of the unit, and (2) nurseclown negotiation of time, space and roles.

\section{Effects of Therapeutic Clowns on the Unit}

Almost unanimously, the participating nurses' responses to the clowns were very positive. They described the clowns as "hilarious" and "phenomenal" and strongly valued their role: "I think that they are just as important as whatever it is I am going to do" (Nurse 3). Nurse participants described how the clowns gave them and the children energy, and 
Table 2. Changes in Physiological Signal Responses from Nurse Participants. ${ }^{a}$

\begin{tabular}{|c|c|c|c|c|c|c|c|c|c|}
\hline \multirow[b]{2}{*}{ ANS signal feature ${ }^{b}$} & \multicolumn{9}{|c|}{ Participant } \\
\hline & 1 & 2 & 3 & 4 & 5 & 6 & 7 & 8 & 9 \\
\hline EDA feature 1 & & & & & & & & & \\
\hline EDA feature 2 & & & & & & & & & \\
\hline Temperature feature & & & & & & & & & \\
\hline Temperature feature & & & & & & & & & \\
\hline Respiration feature 1 & & & & & & & & & \\
\hline Respiration feature 2 & & & & & & & & & \\
\hline Respiration feature 3 & & & & & & & & & \\
\hline Heart feature 1 & & & & & & & & & \\
\hline Heart feature 2 & & & & & & & & & \\
\hline Heart feature 3 & & & & & & & & & \\
\hline
\end{tabular}

${ }^{a}$ Highlighted boxes represent features that exhibited a consistent change (e.g. increase or decrease) from morning to afternoon values on both days when the clowns were present that was opposite to the change from morning to afternoon values when the clowns were absent.

${ }^{\mathrm{b}}$ Feature numbers correspond to the features described in Table 1.

distracted them from problems and stresses: "The kids love it and I think truth be known so do we (laughs) ... it's 10 minutes where you forget what's going on here at work or what's going on in your life and you just sort of chill and laugh" (Nurse 10). According to the interviews, the clowns clearly were a wanted presence on the unit; they helped the nurses and children enjoy the day. This positive effect was most commonly described as a change in the "atmosphere", "mood" or "spirit" of the unit; descriptions included shifts away from

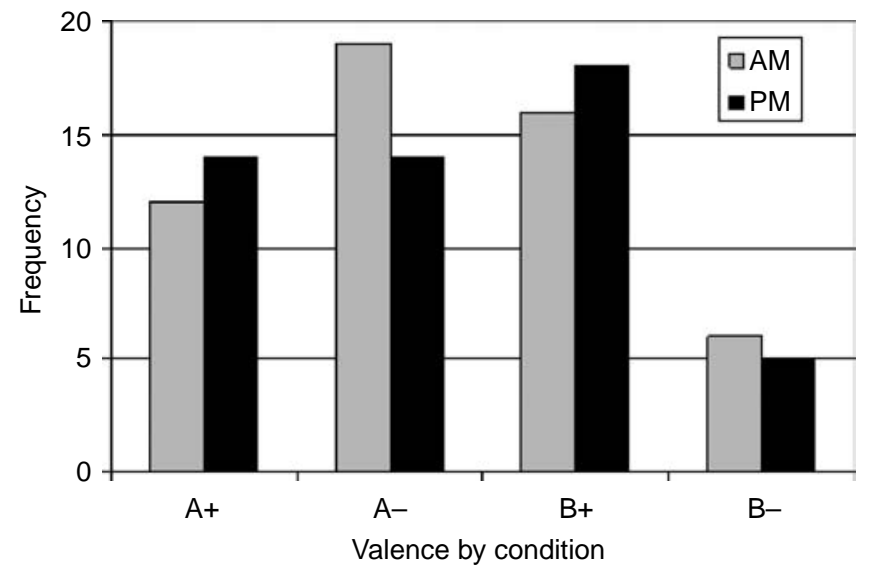

Figure 1. Frequency of nurses' positive $(+)$ and negative $(-)$ valence emotions collapsed across days during morning and afternoon data collection sessions on non-clown (A) and clown days (B). Note: Data missing for two participants for one clown day (B). 
a stressful and anxious atmosphere to one that was lighter, brighter and more enjoyable. While some nurses talked about having direct interactions with the clowns, many referred to a general sense of change in the space of the unit. Nurses said things like: "The staff and the kids gather in a group around the clowns. I think it brings everybody together. It brightens everybody's mood" (Nurse 8) or "I think the whole unit is different when the clowns are on .... It brings up the whole spirit on the unit" (Nurse 12).

The assessment of improved mood seemed to be significantly, though not exclusively, linked to relational factors such as communication and interaction. Nurses described enjoying watching children with the clowns (Nurse 3, Nurse 4) and said they themselves become more playful after spending time in the clowns' presence (Nurse 2). Some described how patients become easier to care for: "These kids are so much easier to work with after they [the clowns] have been here because they are in a better mood. They are so much more pliable and more receptive to what we have to do" (Nurse 3). Some nurses referred to the clowns in pleasant conversation with the children after they had left (Nurse 3 , Nurse 9) or incorporated games that the clowns had used with the children into their nursing care (Nurse 2, Nurse 3).

\section{Negotiation and the Clown's Role}

While nearly all nurses had positive responses to the clowns, it was clear from their descriptions that the clowns' activities were not always simple to integrate with other necessary activities. This was not an unexpected finding, as many nurses - especially those in long-term care settings - face complex and sometimes contradictory expectations about their roles and responsibilities (Abrahamson, 2008; Gilmour, 2005; McGilton et al., 2009). In his description of the challenges of working in a "hybrid" hospital space, Gilmour (2005) notes that long-term care units accommodate both healthcare activities and activities of daily living usually associated with the home. Referring to children's need to play and interact, one nurse described her experience of this tension saying: "You need to know that you can't fulfill that part of their [the children's] human need as well as you want to because you are stretched in so many different directions" (Nurse 4). Some nurses said the clowns changed the way they saw both their roles and their responsibilities for fulfilling hospitalized children's different (and multiple) needs: "It makes me personally look at my profession in a different light. I' $m$ not just there to change a diaper, there to feed a child. We are all here to interact with the children" (Nurse 12).

Sometimes the timing of the clowns' arrival on the unit conflicted with nursing responsibilities necessitating the negotiation of time and space. Nurses described actively choosing to accommodate the clowns within their usual routines in order for the children to have time with them: "Generally when the clowns are here I won't do anything with the [children]. If I can help it, why not let them have ten minutes of nobody poking them or wanting something from them" (Nurse 10). Verbal and tacit negotiation between nurses and clowns sometimes occurred in both directions; nurses deferred their activities or the clowns would return on another occasion. While this negotiation could be seen to increase demands on the nurses, clowns also provided relief for these tensions, as nurses could place some of the responsibility for caring for the children onto their shoulders: "Nurses may do a really great job of looking at health related quality of life but I think sometimes we focus on that and we forget that is not the only component of quality of life, particularly for the kids on [the complex continuing care unit]. My definition of quality of life doesn't just include the functional ability. It also includes being able to participate in life and that's significant. I think the clowns do a really great job of bringing that in" (Nurse 4). 
Finally, the majority of nurses spoke of the value of teamwork. Many made reference to positive collaborations between the nurses and the therapeutic clowns. Nurses who had seen the clowns take part in medical rounds appreciated the way they integrated into the healthcare team. They believed that this integration indicated that the clowns were better informed about the children, were aware of treatment plans, and therefore better prepared for their daily encounters with individual children. This awareness among nurses increased their trust of the professional nature of these non-traditional roles, and strengthened their belief that the clowns had the necessary information to help them negotiate the shared times and spaces of care-giving.

\section{Discussion}

The purpose of this study was to determine the effects of therapeutic clowns interacting with children, on nurses in a pediatric long-term rehabilitation hospital. A combination of physiological, emotional and behavioral measures revealed that indirect exposure to therapeutic clowns had two different types of effects on the nurses: individual and relational. Individual effects were demonstrated in the physiological and self-rated emotion measures: eight of nine nurses experienced consistent changes in their ANS responses on days when the clowns visited the unit in comparison to when the clowns were absent. These findings are in keeping with evidence of physiological change among children exposed to therapeutic clowns (Costa Fernandes \& Arriaga, 2010; Kingsnorth et al., 2010). Changes in the pattern of self-reported moods indicated lower frequencies of negative emotions on clown days. No significant differences in state anxiety were found. The overall mean score was consistent with population norms for working adults (Spielberger, 1983), suggesting that the nurses were not experiencing high levels of stress as part of their daily care activities. Alternatively, secondary exposure to therapeutic clowns may have minimal effects on state anxiety levels; findings are mixed for parents of children undergoing minor surgery (Costa Fernandes \& Arriaga, 2010; Golan et al., 2009; Vagnoli et al., 2005), with only one study reporting a significant reduction (Costa Fernanades \& Arriaga, 2010).

Qualitative, open-ended interview data further revealed that the clowns also affected the unit more broadly. One nurse participant identified the effect of the clowns as cyclical: "I think that its biggest impact on the nurses is not necessarily the direct interaction between the clown and the nurse: the clowns impact on the patient, the patient response affects the nurse. It's a cyclical pattern" (Nurse 4). A few nurses also suggested that the clowns were social catalysts among patients and staff, and that communication among staff improved when the clowns were around: "The interaction is much, much better among staff and patients. I think when they [the clowns] are on the unit it's much, much better ... Sometimes they [the clients] may be sitting by themselves ... But when the clowns are here, then they are involved... instead of being isolated, then they become part" (Nurse 7). These relational effects of therapeutic clowns appeared to be as significant as effects on individuals - suggesting that while some effects of therapeutic clowns can be captured by individual measures, others may impact relationships through communication, role negotiation and the overall meaning of the unit as a social and material space.

\section{Theoretical Understanding of Relational and Spatial Effects}

In order to understand the extension of the impact of therapeutic clowns beyond individuals to affect relationships and the time/space of the unit, we turned to contemporary Health Geography (Andrews, 2004; Martin, Nancarrow, Parker, Phelps \& 
Regen, 2005; Poland et al., 2005; Watson, Murtagh, Lally, Thompson \& McPhail, 2007). This literature demonstrates the importance of the "physical, psychological and social meanings of place" to the experiences and social roles of people inhabiting that place (Martin et al., 2005, p. 1893). Health Geography has gained attention in the last 10 years for foregrounding the significance of social and physical place in healthcare provision (Andrews \& Evans, 2008; Kearns \& Moon, 2002). Its "culture of place" perspective is acknowledged as one of the best ways to grapple with "the complex interrelationship between power, technology, culture and place" found in contemporary healthcare settings (Poland et al., 2005, p. 171).

Clowns typically are understood to disrupt social norms and their presence in hospitals in particular is often perceived as being out of place (Koller \& Gryski, 2008). Experiencing things out of place has been identified in geographic literature as fertile ground for fostering alternative behaviours and meanings to those that are considered normative (Cresswell, 1996). Previous literature has articulated the challenges nurses in long-term care settings identify when they negotiate or try to adhere to rigid definitions of the unit as hospital or home, social space or workplace. While clowns do not eliminate all existing conflicts over time or roles in the healthcare setting, they may buffer some challenges by allowing for flexibility in the meaning and use of spaces. Watson and colleagues (2007) have argued that an awareness of the flexibility of place-based meaning (i.e. being aware that these meanings are socially and relationally constituted) may "help in expanding the therapeutic qualities of particular settings, and support a (more) flexible approach" (p. 865) where alternate paradigms are not necessarily in conflict.

Expanding on the culture of place paradigm is the concept of "health identities", in which "identities are constituted in relation to the social environment that an individual inhabits" (Fox \& Ward, 2008, p. 1007). In this model, identity is not regarded as a fixed state, but rather as a moving, creative and productive state, influenced by the capacity to form new relationships, and shaped by the possibilities and expectations of the environment (Fox \& Ward, 2008). Through this model we can see therapeutic clowns' interventions into the space of the unit as interventions into the process of identity building that is ongoing among all participants. This is seen in the way the clowns contribute to nurses' negotiations of their identities as providers of healthcare and familial, social caring; and in the clowns' impact on the children's identities, shifting them from relatively passive recipients of care to more active participants in games and communication.

Identifying the possibility of a positive impact on the flexibility of the meaning of the healthcare unit and on the identities and relationships of both nurses and children seems to be a good step towards health for all. Particularly in light of the knowledge that negotiation of complex and conflicting roles is one of the greatest stressors for long-term care nurses (Gilmour, 2005) and that children in long-term care settings have many important relationships disrupted - relationships that are central to identity, well-being and life opportunities (Gibson, 2006; Goode, 1984).

\section{Future Directions for Arts-based Intervention Research}

The findings of this exploratory pilot study demonstrate the value of mixed-method approaches for exploring the effects of the arts in healthcare. The relational effects of the therapeutic clowns on the nurses and the unit more broadly could not have been revealed or measured solely using quantitative physiological and behavioural measures. They were captured through open-ended qualitative interviews. Similarly, the depth of responses that were demonstrated by changes in ANS signals would not have been captured through 
interviews or self-report. While previous work on arts-based interventions has alluded to their relational effects - a familiar concept in the social sciences - these effects are challenging to capture within health policy paradigms, which are usually founded on evidence from the natural sciences and the humanities. As diverse fields such as art and health sciences increasingly are integrated, new ways of understanding are required to explain and measure effects across disciplines and between/among individuals.

There has been much discussion about the need for rigorous research to increase the evidence base within the field of the arts in healthcare (Dileo \& Bradt, 2009; Macnaughton et al., 2005; SAH, 2009). The present paper suggests that evaluations of the impact of artsbased interventions should move beyond focusing on individual effects to relational effects, employing measures for social behaviors and system effects. As examples, social behavior measures could include measures of eye contact; looking at faces; emotional expressions; contingent vocalization; joint foci; and 'engaged social interaction' (Kellett, 2005). Larger-scale or systems observations could include measures of noise levels or speed and quality of movement within a healthcare space.

Another avenue for future research may lie in the growing use of complexity theory or the complexity turn in health geography and the social sciences more broadly (Curtis \& Riva, 2010; Gatrell, 2005; Urry, 2005). Although there has been a move to utilize complexity theory in health geography, it is still principally used on large systems (e.g. global systems, large-scale open systems) rather than in smaller systems such as micro spaces (Curtis \& Riva, 2010; Gatrell, 2005). As a relatively new theory for understanding human systems, it is also still lacking empirical applications. Curtis and Riva (2010) suggest that we need to move beyond the theoretical fascination with complexity to develop an empirical application of complexity theory for human systems, arguing that progress in this direction is "gradually being achieved through increased interdisciplinarity in research on health" and "mixed methods, combining intensive, qualitative and interpretative approaches with extensive, quantitative modeling" (p. 220). This paper suggests such theories and methods have the potential to capture the full impact (direct and indirect effects) of arts-based interventions such as therapeutic clowning in healthcare settings.

\section{Conclusions}

This pilot study builds upon an evidence base of positive effects of therapeutic clowns within hospital settings. It reveals that this arts-based intervention also positively impacts healthcare professionals. Participating nurses experienced physiological and self-reported mood changes on days when they witnessed clown-patient interactions or were working when the clowns were present on the unit. In addition to this individual effect, the clowns had a relational effect, enhancing communication between team members, positively affecting nurse-patient relationships, and improving the overall mood and atmosphere of the unit. Mixed-methodology and interdisciplinary theory are encouraged to explore new ways of measuring, understanding, and explaining the effects of arts-based interventions as the fields of art and health become increasingly integrated.

\section{Acknowledgements}

The authors would like to thank Lisa Bonney for her assistance with data collection; Rachel Deutsch for her assistance with a review of the literature; Therapeutic Clowns, Jamie Burnett and Helen Donnelly, for their continued support of this work; and the nurses who contributed to this study. This work was supported by a Bloorview Research Institute Seed Grant [\#08-054]. 


\section{References}

Abrahamson, K. (2008). Role expectations, conflict, and burnout among nursing staff in the long term care setting. Dissertation Abstracts International, A: The Humanities and Social Sciences, 69(09), 3745.

Andrews, G. (2004). (Re)thinking the dynamics between healthcare and place: Therapeutic geographies in treatment and care practices. Area, 36(3), 307-318.

Andrews, G. (2006). Geographies of health in nursing. Health \& Place, 12, 110-118.

Andrews, G., \& Evans, J. (2008). Understanding the reproduction of health care: Towards geographies in health care work. Progress in Human Geography, 36(6), 759-780.

Battrick, C., \& Weaver, K. (2007). Clown humour: The perceptions of doctors, nurses, parents and children. Journal of Children's and Young People's Nursing, 1(4), 174-179.

Beder, J. (2004). Identify Emotions Using Feeling Faces Cards. Retrieved September 12, 2010, from: http://www.feelingfacescards.com

Bertini, M., Isola, E., Paolone, G., \& Curcio, G. (2010). Clowns benefit children hospitalized for respiratory pathologies. Evidenced-based Alternative and Complementary Medicine, doi: 10.1093/ecam/neq064

Blain, S., Chau, T., \& Mihailidis, A. (2008). Peripheral autonomic signals as access pathways for individuals with severe disabilities: A literature appraisal. The Open Rehabilitation Journal, 1(11), 27-37.

Brice, J., \& Barclay, J. (2007). Music eases anxiety of children in cast room. Journal of Pediatric Orthopedics, 27, 831-833.

Chapman, L., Morabito, D., Ladakakos, C., Schreier, H., \& Knudson, M. (2007). The effectiveness of art therapy interventions in reducing post traumatic stress disorder (PTSD) symptoms in pediatric trauma patients. Journal of the American Art Therapy Association, 18, 100-104.

Costa Fernandes, S., \& Arriaga, P. (2010). The effects of clown intervention on worries and emotional responses in children undergoing surgery. Journal of Health Psychology, 15, $405-415$.

Cresswell, T. (1996). In placelout of place: Geography ideology and transgression. Minneapolis: University of Minnesota Press.

Curtis, S., \& Riva, M. (2010). Health geographies I: Complexity theory and human health. Progress in Human Geography, 34(2), 215-223.

Daykin, N., Byrne, E., Soteriou, T., \& O'Connor, S. (2008). The impact of art, design and environment in mental healthcare: A systematic review of the literature. Journal of the Royal Society for the Promotion of Health, 128(2), 85-94.

de Lima, R.A., Azevedo, E.F., Nascimento, L.C., \& Rocha, S.M.M. (2009). A arte do teatro Clown no cuidado as criancas hospitalizadas. Revista de Escola de Enfermagem da USP, 43(1), $186-193$.

Dileo, C., \& Bradt, J. (2009). On creating the discipline, profession, and evidence in the field of arts and healthcare. Arts \& Health: An International Journal for Research, Policy and Practice, 1(2), $168-182$.

Dose, L. (2006). National network for the Arts in Health: Lessons learned from six years of work. The Journal of the Royal Society of the Promotion of Health, 126(3), 110-112.

Fernandes, S.C., \& Arriaga, P. (2010). The effects of clown intervention on worries and emotional responses in children undergoing surgery. Journal of Health Psychology, 15(3), 405-415.

Ferszt, G.G., Massotti, E., Williams, J., \& Miller, J.R. (2000). The impact of an art program on an inpatient oncology unit. Illness, Crisis \& Loss, 8(2), 189-199.

Fox, N.J., \& Ward, K.J. (2008). What are health Identities and how may we study them? Sociology of Health and Illness, 30(7), 1007-1021.

Francani, G.M., Zilioli, D., Silva, P.R., Sant'ana, R.P., \& de Lima, R.A. (1998). Prescription for the day: Infusion of cheer. Using art as an instrument in the care of hospitalized children. Revista latino-americana de enfermagem, 6(5), 27-33.

Francis, S., Willis, J., \& Garvey, A. (2003). Evaluation of the King's Fund's enhancing the healing environment programme. London: The Stationary Office.

Gatrell, A.C. (2005). Complexity theory and geographies of health: A critical assessment. Social Science and Medicine, 60, 2261-2671.

Gatti, M.F.Z., \& da Silva, M.J.P. (2007). Ambient music in the emergency services: The professionals' perception. Revista latino-americana de enfermagem, 15(3), 377-383. 
Gibson, B.E. (2006). Disability, connectivity and transgressing the autonomous body. Journal of Medical Humanities, 27, 187-196.

Gilmour, J.A. (2005). Hybrid space: Constituting the hospital as a home space for patients. Nursing Inquiry, 13(1), 16-22.

Golan, G., Tighe, P., Dobija, N., Perel, A., \& Keidan, I. (2009). Clowns for the prevention of peroperative anxiety in children: A randomized controlled trial. Pediatric Anesthesia, 19, $262-266$.

Gold, C., Solli, H.P., Kruger, V., \& Lie, S.A. (2009). Dose-response relationship in music therapy for people with serious mental disorders: Systematic review and meta-analysis. Clinical Psychology Review, 29, 193-207.

Gold, C., Voracek, M., \& Wigram, T. (2004). Effects of music therapy for children and adolescents with psychopathology: A meta-analysis. Journal of Child Psychology \& Psychiatry, 45, $1054-1063$.

Goode, D.A. (1984). Socially produced identities, intimacy and the problem of competence among the retarded. In L. Barton \& S. Tomlinson (Eds.), Special education and social interests. New York, NY: Nichols.

Goyal, A., \& Keightley, M.L. (2008). Expressive art for the social and community integration of adolescents with acquired brain injuries: A systematic review. Research in Drama Education, 13(3), 337-352.

Higueras, A., Carretero-Dios, H., Munoz, J.P., Idini, E., Ortiz, A., \& Rincon, F., et al. (2006). Effects of a humor-centered activity on disruptive behavior in patients in a general hospital psychiatric ward. International Journal of Clinical and Health Psychology, 6(1), 53-64.

Hilliard, R. (2003). The effects of music therapy on the quality and length of life of people diagnosed with terminal cancer. Journal of Music Therapy, 40, 113-137.

Joinson, C. (1992). Coping with compassion fatigue. Nursing, 22(4), 116-122.

Joronen, K., Rankin, S.H., \& Astedt-Kurki, P. (2008). School-based drama interventions in health promotion for children and adolescents: Systematic review. Journal of Advanced Nursing, 63(2), $116-131$.

KCI Research and Evaluation (2002). Satisfaction and outcome assessment: Hospital artist-inresidence program. (Final Report). The Creative Center arts for people with cancer. Retrieved September 12, 2010, from: http://www.thecreativecenter.org

Kearns, R., \& Moon, G. (2002). From medical to health geography: Novelty, place and theory after a decade of change. Progress in Human Geography, 26(5), 605-625.

Kellett, M. (2005). Catherine's legacy: Social communication development for individuals with profound learning difficulties and fragile life expectancies. British Journal of Special Education, 32(3), 116-121.

Kingsnorth, S., Blain, S., \& Mckeever, P. (2010). Physiological and emotional responses of disabled children to therapeutic clowns: A pilot study. Evidence-Based Complementary and Alternative Medicine, doi:10.1155/2010/neq008

Koller, D., \& Gryski, C. (2008). The life threatened child and the life enhancing clown: Towards a model of therapeutic clowning. Evidence-Based Complementary and Alternative Medicine, 5(1), $17-25$.

Lacey, J.I., \& Lacey, B.C. (1958). Verification and extension of the principle of autonomic responsestereotypy. American Journal of Psychology, 71(1), 50-73.

Linge, L. (2008). Hospital clowns working in pairs - in synchronized communication with ailing children. International Journal of Qualitative Studies on Health and Well-Being, 3(1), 27-38.

Macnaughton, J., White, M., \& Stacy, R. (2005). Researching the benefits of arts in health. Health Education, 105(5), 332-339.

Maratos, A.S., Gold, C., Wang, X., \& Crawford, M.J. (2008). Music therapy for depression. Cochrane Database of Systematic Reviews, 1, doi: 10.1002/14651858.CD004517.pub2

Martin, G.P., Nancarrow, S.A., Parker, H., Phelps, K., \& Regen, E.L. (2005). Place, policy and practitioners: On rehabilitation, independence and the therapeutic landscape in the changing geography of care provision to older people in the UK. Social Science and Medicine, 61(9), $1893-1904$.

McGilton, K.S., Bowers, B., McKenzie-Green, B., Boscart, V., \& Brown, M. (2009). How do charge nurses view their roles in long-term care? The Journal of Applied Gerontology, 28(6), 723-742.

McVicar, A. (2003). Workplace stress in nursing: A literature review. Journal of Advanced Nursing, 44(6), 633-642. 
Oppenheim, D., Simonds, C., \& Hartmann, O. (1997). Clowning on children's wards. Lancet, 350(9094), $1838-1840$.

Phillips, S. (1996). Labouring the emotions: Expanding the remit of nursing work? Journal of Advanced Nursing, 24, 139-143.

Picard, R., Vyzas, E., \& Healey, J. (2001). Toward machine emotional intelligence: Analysis of affective physiological state. IEEE Transactions on Pattern Analysis and Machine Intelligence, 23(10), 1175-1191.

Poland, B., Lehoux, P., Holmes, D., \& Andrews, G. (2005). How place matters: Unpacking technology and power in health and social care. Health and Social Care in the Community, 13(2), 170-180.

Repar, P.A., \& Patton, D. (2007). Stress reduction for nurses through arts-in-medicine at the University of New Mexico Hospitals. Holistic Nursing Practice, 21, 182-186.

Rothieaux, R.L. (1997). The benefits of music in hospital waiting rooms. The Health Care Supervisor, 16, 31-40.

Society for the Arts in Healthcare (SAH) (2009). Arts in healthcare: State of the field report. Washington, DC: Society for the Arts \& Society for the Arts in Healthcare.

Spielberger, C.D. (1983). State-Trait Anxiety Inventory for Adults. Palo Alto: Mind Garden, Inc.

Stuckey, H.L., \& Nobel, J. (2010). The connection between art, healing, and public health: A review of current literature. American Journal of Public Health, 100(2), 254-263.

The Northern Hospital. (2005, June). Humour for good health in the emergency department and child and adolescent health unit. Final report. Retrieved from: http://www.humourfoundation. com.au/page_assets/HumourforGHealth_report.pdf

Treurnicht Naylor, K., Kingsnorth, S., Lamont, A., McKeever, P., \& Macarthur, C. (2010). The effectiveness of music in pediatric healthcare: A systematic review of randomized controlled trials. Evidence-Based Complementary and Alternative Medicine, 2011, doi: 10.1155/2011/464759

Urry, J. (2005). The complexity turn. Theory Culture and Society, 22(5), 1-14.

Vagnoli, L., Caprilli, S., Robiglio, A., \& Messeri, A. (2005). Clown doctors as a treatment for preoperative anxiety in children: A randomized, prospective study. Pediatrics, 116(4), E563-E567.

Walsh, S.M., Martin, S.C., \& Schmidt, L.A. (2004). Testing the efficacy of a creative-arts intervention with family caregivers of patients with cancer. Journal of Nursing Scholarship, $36(3), 214-219$.

Watson, D.B., Murtagh, M.J., Lally, J.E., Thompson, S., \& McPhail, S. (2007). Flexible therapeutic landscapes of labour and the place of pain relief. Health \& Place, 13, 865-876.

Wikstrom, B. (2003). Health professionals' experience of paintings as a conversation instrument: A communication strategy at a nursing home in Sweden. Applied Nursing Research, 16(3), $184-188$.

Wood, M.J., Molassiotis, A., \& Payne, S. (2010). What research evidence is there for the use of art therapy in the management of symptoms in adults with cancer? A systematic review. Psychooncology, doi: 10.1002/pon.1722 